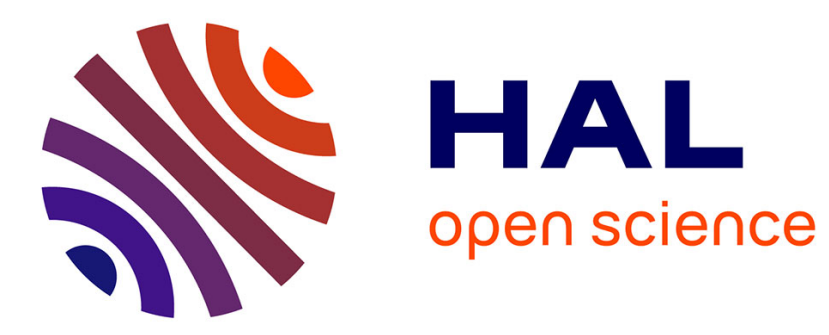

\title{
State-constrained Optimal Control Problems of Impulsive Differential Equations
}

\author{
Nicolas Forcadel, Zhiping Rao, Hasnaa Zidani
}

\section{To cite this version:}

Nicolas Forcadel, Zhiping Rao, Hasnaa Zidani. State-constrained Optimal Control Problems of Impulsive Differential Equations. Applied Mathematics and Optimization, 2013, 68, pp.1-19. 10.1007/s00245-013-9193-5. hal-00653671v2

\section{HAL Id: hal-00653671 \\ https://hal.inria.fr/hal-00653671v2}

Submitted on 3 May 2013

HAL is a multi-disciplinary open access archive for the deposit and dissemination of scientific research documents, whether they are published or not. The documents may come from teaching and research institutions in France or abroad, or from public or private research centers.
L'archive ouverte pluridisciplinaire HAL, est destinée au dépôt et à la diffusion de documents scientifiques de niveau recherche, publiés ou non, émanant des établissements d'enseignement et de recherche français ou étrangers, des laboratoires publics ou privés. 


\title{
STATE-CONSTRAINED OPTIMAL CONTROL PROBLEMS OF IMPULSIVE DIFFERENTIAL EQUATIONS
}

\author{
NICOLAS FORCADEL*, ZHIPING RAO ${ }^{\dagger}$, AND HASNAA ZIDANI ${ }^{\ddagger}$
}

\begin{abstract}
The present paper studies an optimal control problem governed by measure driven differential systems and in presence of state constraints. The first result shows that using the graph completion of the measure, the optimal solutions can be obtained by solving a reparametrized control problem of absolutely continuous trajectories but with time-dependent state-constraints. The second result shows that it is possible to characterize the epigraph of the reparametrized value function by a Hamilton-Jacobi equation without assuming any controllability assumption ${ }^{1}$.
\end{abstract}

AMS Classification. 49J15, 34A37, 34K35, 49Lxx

Keywords. optimal control problems, impulsive differential equations, time-dependent state constraints, time measurable Hamilton-Jacobi equations

1. Introduction . This paper deals with an optimal control problem of measuredriven dynamical systems of the form:

$$
\left\{\begin{array}{l}
d y(t)=g_{0}(t, y(t), \alpha(t)) d t+g_{1}(t, y(t)) d \mu \text { for } t \in(\tau, T] \\
y\left(\tau^{-}\right)=x
\end{array}\right.
$$

where $g_{0}$ and $g_{1}$ are continuous functions whose values, respectively, are in $\mathbb{R}^{d}$ and $\mathcal{M}_{d \times p}$ (the space of $d \times p$ matrices), and $\mu$ is a given vector-valued measure with values in $\mathbb{R}^{p}$ (see section 2 for precise assumptions). The input $\alpha$ is a measurable function belonging to the set of admissible controls $\mathcal{A}$, that is:

$$
\mathcal{A}:=\left\{\alpha:(0, T) \rightarrow \mathbb{R}^{m} \text { measurable function, } \alpha(t) \in A \text { a.e. in }(0, T)\right\},
$$

with $A$ a compact set of $\mathbb{R}^{m}$.

For a given closed subset $\mathcal{K} \subset \mathbb{R}^{d}$, and a final cost function $\varphi: \mathbb{R}^{d} \rightarrow \mathbb{R}^{d}$, the Mayer control problem is:

(1.2) $v(\tau, x):=\inf \left\{\varphi\left(y_{\tau, x}^{\alpha}(T)\right), \quad y_{\tau, x}^{\alpha}\right.$ satisfies $(1.1)$, and $y_{\tau, x}^{\alpha}(t) \in \mathcal{K}$ for $\left.t \in[\tau, T]\right\}$.

Measure-driven dynamical systems arise in many physical or economic applications that undergo forces whose actions have instantaneous effects. These systems are also called impulsive, they include mechanical systems with impacts $[5,12,20,21]$, Faraday waves $[3,22,14]$, and several other applications in biomedicine or neuroscience, see [15] and the references therein.

The impulsive character of the dynamical system (1.1) forces the trajectories to be discontinuous with implicit jumps. The magnitude of this jump should be first clarified in order to well define the behavior of the trajectory at the times of jump

\footnotetext{
* Ceremade, Université Paris-Dauphine, Place du Maréchal de Lattre de Tassigny, F-75775 Paris Cedex 16, France (forcadel@ceremade.dauphine.fr).

${ }^{\dagger}$ Equipe COMMANDS, ENSTA ParisTech \& INRIA-Saclay, 32 Boulevard Victor, F-75015 Paris Cedex, France (Zhiping.Rao@ensta-paristech.fr).

${ }^{\ddagger}$ Equipe COMMANDS, ENSTA ParisTech \& INRIA-Saclay, 32 Boulevard Victor, F-75015 Paris Cedex, France (Hasnaa.Zidani@ensta-paristech.fr).

${ }^{1}$ This work was partially supported by the EU under the 7th Framework Programme Marie Curie Initial Training Network "FP7-PEOPLE-2010-ITN", SADCO project, GA number 264735-SADCO.
} 
and then to have a precise notion of solution. To see this point, consider an example of impulsive ODEs in $4 d$ studied in [15]:

$$
\left\{\begin{array}{l}
d x / d t=\frac{z}{\tau_{r e c}}-\delta\left(t-t_{*}\right) x u \\
d y / d t=-\frac{y}{\tau_{i n}}+\delta\left(t-t_{*}\right) x u \\
d z / d t=\frac{z}{\tau_{i n}}-\frac{z}{\tau_{\text {rec }}} \\
d u / d t=-\frac{u}{\tau_{\text {facil }}}+\delta\left(t-t_{*}\right) k(1-u)
\end{array}\right.
$$

where $\delta(\cdot)$ is the Dirac delta function and $t_{*}$ is a fixed instant. This is a model describing the transit of electrochemical signals between two neurons at a synapse. The signals are passed via neurotransmitters which are stored in vesicles. In this example, $(x, y, z, u)$ represent the quantity of vesicles in different states and $\tau_{\text {rec }}, \tau_{\text {in }}, \tau_{\text {facil }}$ are fixed parameters. The trajectory $Y(\cdot):=(x(\cdot), y(\cdot), z(\cdot), u(\cdot))$ jumps at the time $t_{*}$, then to determine the magnitude of this jump, there are different choices such as $Y\left(t_{*}^{-}\right), Y\left(t_{*}^{+}\right)$and any intermediate value between those two. Besides, since the dimension of this system is larger than 1 , more ambiguity is created when the trajectory jumps in several directions at the same time $t_{*}$ (see [15] for more details).

Several studies have been devoted to the question of giving a precise meaning to the notion of solution of impulsive systems like (1.1) and more generally to defining the product of a measure by a discontinuous function.

An illuminating point of view was introduced and analyzed in a series of papers $[7,8,9,16]$, where the authors used the concept of graph completion to define the multiplication of a point-mass measure with a discontinuous state-dependent term. Basically, we introduce a function $\mathcal{W}:(0, T) \rightsquigarrow(0,1)$ to reparametrize the time variable for the primitive function $B$ of the measure $\mu . \mathcal{W}$ is uniquely determined at each continuity point of $B$, while at the discontinuity points $t_{i}, \mathcal{W}$ is discontinuous and $\left[\mathcal{W}\left(t_{i}^{-}\right), \mathcal{W}\left(t_{i}^{+}\right)\right]$corresponds to a "fictive" time interval (see Figure 1.1). Then
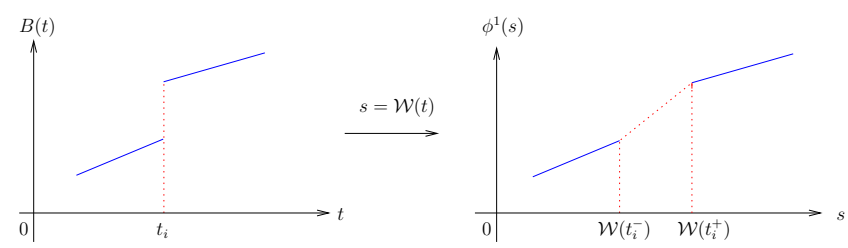

FIG. 1.1. Reparametrization

we consider a graph completion $\left(\phi^{0}, \phi^{1}\right):[0,1] \rightarrow[0, T] \times \mathbb{R}^{p}$ consists of an absolutely continuous map, where $\phi^{0}$ is nondecreasing mapping onto $[0, T]$, and $\phi^{1}$ is an extension to the graph of $B$. When $t \neq t_{i}, \mathcal{W}$ is continuous and

$$
\phi^{0}(s)=t, \phi^{1}(s)=B(t) \text { for } s=\mathcal{W}(t) .
$$

During the fictive time interval $\left[\mathcal{W}\left(t_{i}^{-}\right), \mathcal{W}\left(t_{i}^{+}\right)\right]$, we have

$$
\phi^{0}(s)=t_{i} \Longleftrightarrow s \in\left[\mathcal{W}\left(t_{i}^{-}\right), \mathcal{W}\left(t_{i}^{+}\right)\right]
$$

and the extension part of $\phi^{1}$ prescribes an arc that connects the left and right hand limits of $B$ at the points of discontinuity $t_{i}$.

In the sequel, the set of discontinuities of $B$ will be denoted $\mathcal{T}$. In [16], the solution of (1.1) is defined as solution of an auxiliary differential system reparametrized in time. 
More precisely,

$$
y(t)=z(\mathcal{W}(t)) \quad \text { for } t \in[\tau, T]
$$

where $z$ is solution of

$$
\left\{\begin{array}{l}
\dot{z}(s)=\mathcal{F}(s, z(s), \alpha(s)), \quad s \in(\sigma, 1) \\
z(\sigma)=x
\end{array}\right.
$$

with $\sigma=\mathcal{W}\left(\tau^{-}\right)$, and $\mathcal{F}$ is a measurable function which depends on $g_{0}, g_{1}, \mu$ and on the graph completion $\left(\phi^{0}, \phi^{1}\right)$ (the precise expression of $\mathcal{F}$ will be given in Section $2)$. The reparametrized solution $z$ of $(1.3 \mathrm{~b})$ is continuous and is well defined on the reparametrized time interval. In this way the multiplication of $g_{1}(y(t))$ by $\mu$ in the jump points is unambiguously defined.

In [16], a natural graph completion is introduced and analyzed. It consists on connecting the endpoints of the jumps of $B$ by a straight line. This graph completion is said to be in the canonical form and it has been proved to lead to the same measuresolution given by the integral form:

$$
y(t)=x+\int_{\tau}^{t} g_{0}(s, y(s), \alpha(s)) d s+\int_{[\tau, t]} g_{1}(s, y(s)) d \mu .
$$

Of course, the above integral form has also to be well defined. It is known that each graph completion may lead to a different solution [6]. Further properties of the Graph completion concept and generalization to measure driven differential inclusions can be found in $[25,26,31,30]$.

In the present paper, the solution of (1.1) will be defined by using the canonical graph completion. For the convenience of the reader, the concept of canonical graph completion and the notion of measure-solution are briefly recalled in Section 2.

With a precise definition of trajectories in hand, we can study the control problem (1.2). Let us mention that several works have carried out on the necessary optimality conditions for problem (1.2) [27, 2]. The present paper focuses mainly on the characterization of the value function $v$ using the HJB approach. The main difficulties lie in the presence of the measure $\mu$ and of the state constraints.

It is easy to see that the value function $v$ satisfies a Dynamic Programming Principle (DPP) which formally yields the following HJB equation:

$$
\left\{\begin{array}{l}
-v_{t}(t, x)+\sup _{a \in \mathcal{A}}\left\{-D v(t, x) \cdot\left(g_{0}(t, x, a)+g_{1}(t, x) d \mu\right)\right\}=0, \\
v(T, x)=\varphi(x) .
\end{array}\right.
$$

However, it is not clear in what sense the term " $D v \cdot d \mu$ " should be understood since there is no viscosity notion for this HJB equation with the measure term. In order to overcome this problem, using the concept of graph completion, one can consider a reparameterized optimal control problem where the new value function $\bar{v}$ is defined by:

(1.6) $\bar{v}(\sigma, x)=\inf _{\alpha \in \mathcal{A}}\left\{\varphi\left(z_{\sigma, x}^{\alpha}(1)\right), z_{\sigma, x}^{\alpha}\right.$ satisfies $(1.3)$, and $z_{\sigma, x}^{\alpha}(s) \in \mathcal{K}$ in $\left.(\sigma, 1)\right\}$.

This problem is now classical and the characterization of $\bar{v}$ by a HJB equation falls into the already known theory. Moreover, when $\mathcal{K}$ is the hole space $\mathbb{R}^{d}$ (no state 
constraints), it has been shown in [11] that the value function of the original problem (1.2) can be obtained by:

$$
v(\tau, x)=\bar{v}(\mathcal{W}(\tau), x)
$$

This relation is no more true when the control problem is in presence of state constraints (when $\mathcal{K} \neq \mathbb{R}^{d}$ ). Actually, as said before, by the graph-completion technics, to each trajectory $y$ of the problem (1.1) correspond a trajectory $z$ solution to the reparametrized system (1.3). However, it may happen that the trajectory $y$ satisfies the state constraints while the trajectory $z$ does not. Indeed, $y$ and $z$ coincide only on the branches of continuity of $y$. On these branches the state constraints should be satisfied for both $y$ and $z$. However, $z$ has also other branches corresponding to the fictive time intervals and it may happen that the state constraints fail to be satisfied on these intervals, unless some controllability assumptions are satisfied, see [17].

In the general case, it is more natural to consider the auxiliary control problem in the form of

(1.7) $\bar{v}(\sigma, x)=\inf _{\alpha \in \mathcal{A}}\left\{\varphi\left(z_{\sigma, x}^{\alpha}(1), z_{\sigma, x}^{\alpha}\right.\right.$ satisfies $(1.3)$, and $z_{\sigma, x}^{\alpha}(s) \in \mathbb{K}_{s}$ in $\left.\left(\sigma^{-}, 1\right)\right\}$,

where $\mathbb{K}_{s}=\mathcal{K}$ for $s=\mathcal{W}(t)$ with $t \in[0, T] \backslash \mathcal{T}$ and $\mathbb{K}_{s}=\mathbb{R}^{d}$ (or any other big set containing all the trajectories) for $s \in \cup_{t_{i} \in \mathcal{T}}\left[t_{i}^{-}, t_{i}^{+}\right]$.

Hamilton-Jacobi approach for state-constrained control problems have been extensively studied in the literature $[28,29,13,18,4]$. When the state constraints are time-dependent, the characterization of the value function becomes more complicated [19].

The main idea to treat the time-dependent state constraints is to characterize the epigraph of the value function instead of characterizing the value function directly. Here, we extend the ideas developed in [1] to the case of time-dependent state constraints, and prove that the epigraph of $\vartheta$ can be characterized by means of a Lipschitz continuous viscosity solution of a time-measurable HJB equation (this notion of viscosity notion will be made precise in Section 4).

The paper is organized as follows. In section 2, the control problem is described and the notion of solution for the state equation is recalled. Section 3 is devoted to the study of the reparametrized control problem while the main characterization of the value function of the auxiliary control problem is given in Section 4 .

Notations. For each $r>0, x \in \mathbb{R}^{d}$ we will denote by $B_{r}(x)$ the closed ball of radius $r$ centered in $x$.

For a function $f:[a, b] \rightarrow \mathbb{R}^{d}$ we denote by $V_{a}^{b}(f)$ the total variation of $f$ on $[a, b]$ and by $B V\left([a, b] ; \mathbb{R}^{d}\right)$ the set of functions $f:[a, b] \rightarrow \mathbb{R}^{d}$ with bounded total variation on $[a, b]$.

In the sequel, we use the notations: $f\left(t^{+}\right):=\lim _{s \rightarrow t^{+}} f(s), f\left(t^{-}\right):=\lim _{s \rightarrow t^{-}} f(s)$ and $[f]_{t}:=f\left(t^{+}\right)-f\left(t^{-}\right)$. And finally, we denote by $A C\left([a, b] ; \mathbb{R}^{d}\right)$ the set of absolutely continuous functions from $[a, b]$ to $\mathbb{R}^{d}$.

2. State constrained problems with BV trajectories. In this section, we formulate a state-constrained control problem with discontinuous trajectories. Then, we recall the graph completion technics and the definition of solution for the state equation introduced in $[7,16]$. 
2.1. The state equation and the graph completion technique. Let $T$ be a fixed final time, $x \in \mathcal{K}$ be an initial position. Given a Radon measure $\mu$ and a control variable $\alpha \in \mathcal{A}$, we consider the controlled trajectory $y_{x, \tau}^{\alpha}(t): \mathbb{R}^{+} \rightarrow \mathbb{R}^{d}$ solution of

$$
\left\{\begin{array}{l}
d y(t)=g_{0}(t, y(t), \alpha(t)) d t+g_{1}(t, y(t)) d \mu \text { for } t \in(\tau, T] \\
y\left(\tau^{-}\right)=x
\end{array}\right.
$$

where $\alpha$ belongs to the set $\mathcal{A}$ of admissible controls, given by:

$$
\mathcal{A}:=\left\{\alpha:(0, T) \rightarrow \mathbb{R}^{m} \text { measurable function, } \alpha(t) \in A \text { a.e. in }(0, T)\right\},
$$

with $A$ a compact set of $\mathbb{R}^{m}$. The functions $g_{0}$ and $g_{1}$ will be assumed to satisfy:

(Hg1) $g_{0}:(0, T) \times \mathbb{R}^{d} \times A \rightarrow \mathbb{R}^{d}$ and $g_{1}:(0, T) \times \mathbb{R}^{d} \rightarrow \mathcal{M}_{d \times p}$ are measurable functions with respect to the time variable and are continuous with respect to the other variables. Moreover, for any $y \in \mathbb{R}^{d}$ and any $a \in A, g_{0}(\cdot, y, a) \in$ $L^{1}(0, T)$ and $g_{1}(\cdot, y) \in L_{\mu}^{1}(0, T)$.

(Hg2) $\exists k_{0}>0$ such that $\forall y, z \in \mathbb{R}^{d}, a \in A, t \in \mathbb{R}^{+}$, we have:

$$
\left|g_{0}(t, y, a)-g_{0}(t, z, a)\right|+\left|g_{1}(t, y)-g_{1}(t, z)\right| \leq k_{0}|y-z| .
$$

$\left|g_{0}(t, y, a)\right| \leq L_{g}$ and $\left|g_{1}(t, y)\right| \leq L_{g}, \forall y \in \mathbb{R}^{d}, a \in A$, and for a.e. $t \in \mathbb{R}^{+}$.

Moreover, for a.e $t \in(0, T)$ and for every $x \in \mathbb{R}^{d}, g_{0}(t, x, A)$ is a convex set.

The state equation (1.1) is described by a driven-measure differential system, and as mentioned in the introduction, the jumps of the solution should be well described in order to define unambiguous notion of solution. Here we adapt the definition introduced in $[7,16]$. Let $B$ be the left continuous primitive of $\mu$, i.e.

$$
B(t)=\mu([0, t)),
$$

then $B \in B V\left([0, T] ; \mathbb{R}^{p}\right)$ and its distributional derivative $\dot{B}$ coincides with $\mu$ on $[0, T)$. Consider also $\mathcal{T}:=\left\{t_{i}, i \in \mathcal{I}\right\}$ the set of all the discontinuity points of $B$, where $\mathcal{I}$ is the at most countable index of these discontinuity points.

Furthermore, let $\left\{\psi_{t}\right\}_{t \in \mathcal{T}}$ be a family of linear maps from $[0,1]$ into $\mathbb{R}^{M}$ such that

$$
\psi_{t_{i}}(\sigma):=B\left(t_{i}^{-}\right)+\sigma\left(B\left(t_{i}^{+}\right)-B\left(t_{i}^{-}\right)\right), \text {for } \sigma \in[0,1], i \in \mathcal{I} \text {. }
$$

Each $\psi_{t_{i}}$ joins $B\left(t_{i}^{-}\right)$to $B\left(t_{i}^{+}\right)$. We will denote by $\xi$ the solution of:

$$
\frac{d \xi(\sigma)}{d \sigma}=g_{1}(\sigma, \xi(\sigma)) \frac{d \psi_{t}(\sigma)}{d \sigma}, \text { for } \sigma \in(0,1], \xi(0)=\bar{\xi},
$$

and we set $\xi\left(\bar{\xi}, \psi_{t}\right):=\xi(1)-\bar{\xi}$. Now, we are ready to state the definition of solution introduced by Dal Maso and Rampazzo in [16].

DEFINITION 2.1. Fix an initial position and time $(\tau, x)$ and a control variable $\alpha \in \mathcal{A}$, the function $y_{x, \tau}^{\alpha} \in B V\left([\tau, T] ; \mathbb{R}^{d}\right)$ is a solution of $(2.1)$ if for each Borel subset $\mathcal{B}$ of $] \tau, T[$ we have

$$
\int_{\mathcal{B}} d y(t)=\int_{\mathcal{B}} g_{0}(t, y(t), \alpha(t)) d t+\int_{\mathcal{B} \backslash \mathcal{T}} g_{1}(t, y(t)) d \mu+\sum_{t_{i} \in \mathcal{T}} \xi\left(y\left(t_{i}^{-}\right), \psi_{t_{i}}\right)
$$


and $y\left(\tau^{-}\right)=x$. Moreover, if $\tau \in \mathcal{T}$ we have $y\left(\tau^{+}\right)=\xi\left(x, \psi_{\tau}\right)$.

Remark 2.2. Here for simplicity, we have considered $\left\{\psi_{t}\right\}_{t \in \mathcal{T}}$ as linear maps. In fact, $\left\{\psi_{t}\right\}_{t \in \mathcal{T}}$ can be any family of Lipschitz continuous maps from $[0,1]$ into $\mathbb{R}^{M}$ with each $\psi_{t}$ joining $B(t)$ to $B\left(t^{+}\right)$. But we also point out that a different choice of $\left\{\psi_{t}\right\}_{t \in \mathcal{T}}$ leads to a different definition of solution for (2.1).

This definition gives a precise notion for the solution of the equation (1.1). Recall now another definition based on the graph completion technique and which leads to a characterization of the solution through the unique absolutely continuous solution of a reparametrized system. In order to do that, we define $\mathcal{W}:[0, T] \rightarrow[0,1]$ as follows:

$$
\mathcal{W}(t)=\frac{t+V_{0}^{t}(B)}{T+V_{0}^{T}(B)}, \text { for } t \in[0, T]
$$

then $\mathcal{W}$ is continuous on $[0, T] \backslash \mathcal{T}$. The canonical graph completion of $B$ corresponding to the family of linear functions $\left(\psi_{t}\right)_{t \in \mathcal{T}}$ is then defined by:

$$
\begin{aligned}
\Phi(s) & =\left(\phi^{0} ; \phi^{1}\right)(s) \\
& = \begin{cases}(t ; B(t)) & \text { if } s=\mathcal{W}(t), t \in[0, T] \backslash\left\{t_{1}, \ldots, t_{M}\right\} \\
\left(t_{i} ; \psi_{t_{i}}\left(\frac{s-\mathcal{W}\left(t_{i}\right)}{[\mathcal{W}]_{t_{i}}}\right)\right) & \text { if } s \in\left[\mathcal{W}\left(t_{i}\right), \mathcal{W}\left(t_{i}^{+}\right)\right], t_{i} \in \mathcal{T},\end{cases}
\end{aligned}
$$

where

$$
\psi_{t_{i}}\left(\frac{s-\mathcal{W}\left(t_{i}\right)}{[\mathcal{W}]_{t_{i}}}\right)=B\left(t_{i}\right)+\frac{[B]_{t_{i}}}{[\mathcal{W}]_{t_{i}}}\left(s-\mathcal{W}\left(t_{i}\right)\right)
$$

Following [16], we introduce the reparametrized system defined by:

$$
\left\{\begin{aligned}
\frac{d z}{d s}(s)= & g_{0}\left(\phi^{0}(s), z(s), \alpha\left(\phi^{0}(s)\right)\right) \frac{d \phi^{0}}{d s}(s)+ \\
& g_{1}\left(\phi^{0}(s), z(s)\right)\left(\mu^{a}\left(\phi^{0}(s)\right) \frac{d \phi^{0}}{d s}(s)+\frac{d \phi^{1}}{d s}(s)\right) \quad \text { for } s \in(\sigma, 1], \\
z(\sigma)= & x
\end{aligned}\right.
$$

where $\sigma:=\mathcal{W}(\tau), \mu^{a}$ is the absolutely continuous part of the measure $\mu$ with respect to the Lebesgue measure, i.e. $\mu(t)=\mu^{a}(t) d t+\mu^{s}$. We note that the derivatives of $\phi^{0}, \phi^{1}$ are measurable functions. Therefore, under assumptions (Hg1)-(Hg3), the Caratheodory system (2.9) has a unique solution and according to [11, Theorem 2.2]), the following holds.

Proposition 2.3. Assume (Hg1)-(Hg3), then $y_{x, \tau}^{\alpha} \in B V\left([\tau, T] ; \mathbb{R}^{d}\right)$ is a solution of (2.1) (in the sense of Definition 2.1) if and only if there exists a solution $z_{x, \sigma}^{\alpha} \in A C\left([\sigma, 1] ; \mathbb{R}^{d}\right)$ of $(2.9)$ such that

$$
z_{x, \sigma}^{\alpha}(\mathcal{W}(t))=y_{x, \tau}^{\alpha}(t), \quad \forall t \in[\tau, T] .
$$

The proof uses the same arguments introduced in [16, Theorem 2.2] for the Lipschitz continuous trajectories. The main difference here is to deal with the absolutely continuous trajectories which are less regular than Lipschitz arcs. To overcome this difficulty, we use a generalized chain rule for the composition of absolutely continuous functions and BV functions (presented in [10]).

The statement of proposition 2.3 links each BV trajectory solution of (2.1) with an absolutely continuous function satisfying the parametrized equation (2.9). 
2.2. The control problem. For a given measure $\mu$ and a given corresponding graph completion $\left(\phi^{0}, \phi^{1}\right)$, consider the set of BV trajectories satisfying (2.1):

$S_{[\tau, T]}(x):=\left\{y=y_{\tau, x}^{\alpha}, y\right.$ satisfies $(2.1)$ in the sense of Definition 2.1 and $\left.\alpha \in \mathcal{A}\right\}$, and the set of reparametrized trajectories:

$$
S_{[\sigma, 1]}^{P}(x):=\left\{z=z_{\sigma, x}^{\alpha} \text { satisfies }(2.9) \text { and } \alpha \in \mathcal{A}\right\} .
$$

Given a closed subset $\mathcal{K} \subset \mathbb{R}^{d}$ and a final cost function $\varphi: \mathbb{R}^{d} \rightarrow \mathbb{R}^{d}$, the Mayer control problem governed by the impulse systems is:

$$
v(\tau, x):=\inf \left\{\varphi(y(T)), y \in S_{[\tau, T]}(x), \text { and } y(t) \in \mathcal{K} \text { for } t \in[\tau, T]\right\} .
$$

We assume in the sequel that:

$(\mathbf{H} \varphi) \varphi: \mathbb{R}^{d} \rightarrow \mathbb{R}$ is a Lipschitz continuous function.

It is easy to prove that the value function satisfies a classic Dynamic Programming Principle (see [24] for a general DPP). For each $\tau \in] 0, T[$, and every $h \in[0, T-\tau]$, we have

$$
\begin{aligned}
& v(\tau, x)=\inf _{\alpha \in \mathcal{A}} v\left(\tau+h, y_{x, \tau}^{\alpha}(\tau+h)\right) \quad \text { for } x \in \mathcal{K}, \\
& v(\tau, x)=+\infty, \quad \text { for } x \notin \mathcal{K} .
\end{aligned}
$$

According to this DPP, we can formally derive the HJB equation:

$$
\begin{cases}-v_{t}(t, x)+H(t, x, D v(t, x))=0 & \text { for }(t, x) \in(0, T) \times \mathcal{K}, \\ v(T, x)=\varphi(x) & \text { for } x \in \mathcal{K}\end{cases}
$$

where the Hamiltonian is

$$
H(t, x, p)=\sup _{\alpha \in \mathcal{A}}\left\{-p \cdot\left(g_{0}(t, x, a)+g_{1}(t, x) \mu\right)\right\} .
$$

However, this equation is just formal and several difficulties arise when characterizing the value function by a HJB equation. The main difficulty comes from the fact that in general the value function is not $C^{1}$ and it is not clear in which sense the $D v \cdot \mu$ should be understood. The second difficulty comes from the fact that the control problem is in presence of state constraints.

To deal with these difficulties, the idea would be to consider the reparametrized control problem instead of $(2.12)$ (for $\sigma=\mathcal{W}(\tau)$ ):

$$
\bar{v}(\sigma, x):=\inf \left\{\varphi(z(1)), z \in S_{[\sigma, 1]}^{P}, z(s) \in \mathcal{K} \text { for } s \in[\sigma, 1]\right\} .
$$

When the control problem is without state constraints (ie, when $\mathcal{K} \neq \emptyset$ ), we have (see [11]):

$$
v(\tau, x)=\bar{v}(\mathcal{W}(\tau), x) \quad \text { for any } x \in \mathbb{R}^{d}, \tau \in(0, T) .
$$

However, this relation may not be valid when the problem is in presence of state constraints (ie, when $\mathcal{K} \neq \mathbb{R}^{d}$ ). The reason is that even if an admissible trajectory $y$ stays in $\mathcal{K}$ in $[\tau, T]$, it may happen that the reparametrized trajectory leave $\mathcal{K}$ during the "fictive" time intervalles $s \in\left[\mathcal{W}\left(t_{i}\right), \mathcal{W}\left(t_{i}^{+}\right)\right]$, where $t_{i}$ is a discontinuous point of $\mathcal{W}$. 


\section{Reparametrized control problem.}

3.1. Case when some controllability assumptions are satisfied. In this section, we investigate some assumptions that can ensure that (2.15) holds. Here, more assumptions on the behavior of $g_{1}$ at the boundary of $\mathcal{K}$ are needed:

$$
g_{1}(t, x) \in T_{\mathcal{K}}(x) \quad \text { for any } t \in(0,1) \text {, and } x \in \mathcal{K},
$$

where $T_{\mathcal{K}}(x)$ is the tangent cone of $\mathcal{K}$ at $x$ defined by

$$
T_{\mathcal{K}}(x):=\left\{p \in \mathbb{R}^{d} \mid \exists t_{0}, \forall t \in\left(0, t_{0}\right), x+t p+o(t) \in \mathcal{K}\right\} .
$$

This assumption states that for any admissible trajectory $y$ which lies in $\mathcal{K}$ before a jump (ie, $y\left(t_{i}^{-}\right) \in \mathcal{K}$ ) will stay in $\mathcal{K}$ also after the jump (ie, $y\left(t_{i}^{+}\right) \in \mathcal{K}$ ). Moreover, the following holds, see [17]:

THEOREM 3.1. Assume that (Hg1)-(Hg3) are satisfied and that the condition (3.1) holds. Then, for every $x \in \mathcal{K}$, and $\tau \in(0, T)$ :

(i) to each admissible trajectory $y \in S_{[\tau, T]}(x)$ satisfying the pointwise constraints $y(s) \in \mathcal{K}$ corresponds a trajectory $z \in S_{\left[\mathcal{W}\left(\tau^{-}\right), 1\right]}^{P}(x)$ satisfying also the same constraints $z(s) \in \mathcal{K}$, where $\mathcal{W}$ is given by $(2.6)$.

(ii) Moreover, assume $(H \varphi)$. Let $v$ and $\bar{v}$ be defined respectively by (2.11) and (2.14). Therefore,

$$
v(\tau, x)=\bar{v}(\mathcal{W}(\tau), x)
$$

According to this theorem, the characterization of $v$ can be obtained through the reparametrized value function $\bar{v}$. The latter is associated to a control problem of continuous trajectories. We refer to [17] for further discussion on the HJB equation satisfied by $\bar{v}$ and turn now our attention to the more general case when (3.1) does not necessarily hold.

3.2. General case without any controllability assumption. Here no additional condition is made on the vector field $g_{1}$ on the boundary of $\mathcal{K}$. The first aim would be to find a more convenient auxiliary control problem for which the value function will coincide with the original function $v$. From the discussion of the previous section, it turns out that the state constraints should be somehow relaxed for the reparametrized trajectories during the "fictive" time intervals $\left[\mathcal{W}\left(t_{i}^{-}\right), \mathcal{W}\left(t_{i}^{+}\right)\right]$. For this, time-dependent state constraints in the form of $z(s) \in \mathbb{K}(s)$ should be considered with $\mathbb{K}(s)$ equal to $\mathcal{K}$ when $s=\mathcal{W}(t)$ for any $t \in[0, T] \backslash \mathcal{T}$, and $\mathbb{K}(s)$ is large enough for any $\left.s \in \bigcup_{t_{i} \in \mathcal{T}}\right] \mathcal{W}\left(t_{i}^{-}\right), \mathcal{W}\left(t_{i}^{+}\right)$[ so that the constraints are satisfied by any reparametrized trajectory without assuming any viability conditions like (3.1).

In the sequel, we will use the notation:

$$
\bar{s}_{i}^{ \pm}=\mathcal{W}\left(t_{i}^{ \pm}\right) \text {for every } t_{i} \in \mathcal{T},
$$

where $\mathcal{T}$ is the set of discontinuity points of $B$. We have the following theorem:

THEOREM 3.2. Assume (Hg1)-(Hg3) and assume $\mathcal{K}$ to be a closed subset of $\mathbb{R}^{d}$. Consider the set-valued map $x \rightsquigarrow \mathbb{K}(x)$ defined by:

$$
\mathbb{K}(s)= \begin{cases}\mathcal{K} & \text { if } s=\mathcal{W}(t), t \in[0, T] \backslash \mathcal{T}, \\ \mathcal{K}+B\left(0, L_{g} \delta\left(s-\bar{s}_{i}^{-}\right)\right) & \text {if } s \in\left[\bar{s}_{i}^{-}, \frac{\bar{s}_{i}^{-}+\bar{s}_{i}^{+}}{2}\right], \\ \mathcal{K}+B\left(0, L_{g} \delta\left(\bar{s}_{i}^{+}-s\right)\right) & \text { if } s \in\left[\frac{\bar{s}_{i}^{-}+\bar{s}_{i}^{+}}{2}, \bar{s}_{i}^{+}\right],\end{cases}
$$


with $L_{g}$ defined in (Hg2) and $\delta=T+V_{0}^{T}(B)$. Then the multi-application $\mathbb{K}$ is upper semicontinuous (usc, in short)

(ii) Moreover, if we define

$$
\bar{v}(\sigma, x)=\inf _{\alpha \in \mathcal{A}}\left\{\varphi\left(z_{x, \sigma}^{\alpha}(1)\right), z_{x, \sigma}^{\alpha} \quad \text { solution of }(2.9) \text { and } z_{x, \sigma}^{\alpha}(s) \in \mathbb{K}(s), \forall s \in[\sigma, 1]\right\} \text {, }
$$

then we have:

$$
v(\tau, x)=\bar{v}(\mathcal{W}(\tau), x) \quad \text { for every } \tau \in[0, T]
$$

Proof. To prove assertion (i), we claim that for any $t \in\left[\bar{s}_{i}^{-}, \frac{\bar{s}_{i}^{-}+\bar{s}_{i}^{+}}{2}\right]$ (resp. $t \in$ $\left[\frac{\bar{s}_{i}^{-}+\bar{s}_{i}^{+}}{2}, \bar{s}_{i}^{+}\right]$), and $s \in\left[\bar{s}_{i}^{-}, t\right]$ (resp. $s \in\left[t, \bar{s}_{i}^{+}\right]$), then we have

$$
\operatorname{dist}\left(\mathbb{K}(s), \mathbb{K}(t)^{c}\right) \leq L_{g} \delta|t-s| .
$$

Consider first the case when $t \in\left[\bar{s}_{i}^{-}, \frac{\bar{s}_{i}^{-}+\bar{s}_{i}^{+}}{2}\right]$ and assume that for any $x \in \partial \mathbb{K}(s)$ and $y \in \partial \mathbb{K}(t)$, the following holds:

$$
\|x-y\|>L_{g} \delta(t-s) .
$$

Let $y_{0} \in \partial \mathbb{K}(t)$ and set $z_{0} \in P_{\mathcal{K}_{i}}\left(y_{0}\right)$. By the definition of $\mathbb{K}$, we deduce that

$$
\left\|y_{0}-z_{0}\right\|=L_{g} \delta\left(t-\bar{s}_{i}^{-}\right) .
$$

Let $x_{0} \in\left[y_{0}, z_{0}\right] \cap \partial \mathbb{K}(s)$. We then have

$$
\begin{aligned}
\left\|x_{0}-z_{0}\right\| & =\left\|y_{0}-z_{0}\right\|+\left\|y_{0}-x_{0}\right\| \\
& <L_{g} \delta\left(t-\bar{s}_{i}^{-}\right)-L_{g} \delta(t-s) \\
& =L_{g} \delta\left(s-\bar{s}_{i}^{-}\right)
\end{aligned}
$$

which contradicts the fact that $x_{0} \in \partial \mathbb{K}(s)$.

To prove assertion (ii), let us consider some $z_{x, \sigma}^{\alpha}$ solution of (2.9) and satisfying that $z_{x, \sigma}^{\alpha}(s) \in \mathbb{K}(s), \forall s \in[\sigma, 1]$. For any $t \in[\tau, T]$ and $s=\mathcal{W}(t)$, we have $\mathbb{K}(s)=\mathcal{K}$ which implies

$$
z_{x, \sigma}^{\alpha}(\mathcal{W}(t)) \in \mathcal{K}
$$

On the other side, consider some $z_{x, \sigma}^{\alpha}$ solution of $(2.9)$ and satisfying that $z_{x, \sigma}^{\alpha}(\mathcal{W}(t)) \in$ $\mathcal{K}, \forall t \in[\tau, T]$. We want to prove that $z_{x, \sigma}^{\alpha}(s) \in \mathbb{K}(s), \forall s \in[\sigma, 1]$. If $s=\mathcal{W}(t)$ for some $t \in[\tau, T] \backslash \mathcal{T}, \mathbb{K}(s)=\mathcal{K}$ and the result is obvious. If $s \in\left[\bar{s}_{i}^{-}, \frac{\bar{s}_{i}^{-}+\bar{s}_{i}^{+}}{2}\right]$, since the dynamic of $z_{x, \sigma}^{\alpha}$ is bounded by $L_{g} \delta$, we have

$$
\left|z_{x, \sigma}^{\alpha}(s)-z_{x, \sigma}^{\alpha}\left(\bar{s}_{i}^{-}\right)\right|<L_{g} \delta\left(s-\bar{s}_{i}^{-}\right)
$$

and we know that $z_{x, \sigma}^{\alpha}\left(\bar{s}_{i}^{-}\right) \in \mathcal{K}$, then

$$
\operatorname{dist}\left(z_{x, \sigma}^{\alpha}(s), \mathcal{K}\right)<L_{g} \delta\left(s-\bar{s}_{i}^{-}\right),
$$

which implies that

$$
z_{x, \sigma}^{\alpha}(s) \in \mathbb{K}(s)
$$


In the case of $s \in\left[\frac{\bar{s}_{i}^{-}+\bar{s}_{i}^{+}}{2}, \bar{s}_{i}^{+}\right]$, the argument is quite similar by considering a backward dynamical system and using the fact that $z_{x, \sigma}^{\alpha}\left(\bar{s}_{i}^{+}\right) \in \mathcal{K}$. We conclude that

$$
y(t) \in \mathcal{K} \text { for } t \in[\tau, T] \Leftrightarrow z(s) \in \mathbb{K}(s) \text { for } s \in[\sigma, 1]
$$

Then (3.5) follows by the fact that $y_{x, \tau}^{\alpha}(T)=z_{x, \mathcal{W}(\tau)}^{\alpha}(\mathcal{W}(T))=z_{x, \sigma}^{\alpha}(1)$ and the definitions of $v$ and $\bar{v}$.

Theorem 3.2 suggests to compute first the new auxiliary value function and then deduce the original value function $v$ by the formula (3.5). The auxiliary reparametrized control problem is in presence of time-dependent state constraints. Recall that several papers have been devoted to study the characterization of the value function for state constrained control problems. Under some controllability assumption and when the set of state-constraints is not time-dependent, the value function can be shown to be the unique constrained-viscosity solution on an adequate HJB equation, see in $[28,29,18]$. We refer also to $[4,1]$ for a discussion on the general case where the control problem is lacking controllability properties.

Here the control problem (3.4) is in presence of time-dependent state constraints and no controllability assumption is assumed. The characterization of $\bar{v}$ by an HJB equation on a tube $\mathbb{K}$. is not a simple task because the evolution of $\bar{v}$ depends also on the evolution of the map $\mathbb{K}$. Here we extend to time-dependent state-constrained control problems an idea developed recently in [1] which allows to compute all the epigraph of the value function $\bar{v}$ by solving an appropriate variational HJB equation.

4. Optimal control problems with time-dependent state constraints. In this section, the main result concerns optimal control problems with time-dependent state constraints and time-measurable Hamiltonians. Introduce the function $\mathcal{F}$ defined by:

$$
\mathcal{F}(s, z, a)=g_{0}\left(\phi^{0}(s), z, a\right) \dot{\phi}^{0}(s)+g_{1}\left(\phi^{0}(s), z\right)\left(\mu^{a}\left(\phi^{0}(s)\right) \dot{\phi}^{0}(s)+\dot{\phi}^{1}(s)\right),
$$

where $\phi^{0}$ and $\phi^{1}$ are given in (2.7).

Remark 4.1. All the results of this section hold in a more general setting, where the following time-dependent state constrained Mayer's control problem is considered:

$$
\vartheta(\sigma, x)=\inf _{\alpha \in \mathcal{A}}\left\{\varphi\left(z_{x, \sigma}^{\alpha}(1)\right), z_{x, \sigma}^{\alpha} \quad \text { solution of }(4.1) \text { and } z_{x, \sigma}^{\alpha}(\theta) \in \mathbb{K}_{\theta}, \forall \theta \in[\sigma, 1]\right\} \text {. }
$$

with the convention that $\inf \emptyset=+\infty$, where the state equation is given by:

$$
\left\{\begin{array}{l}
\dot{z}(s)=\mathcal{F}(s, z(s), \alpha(s)), \quad \text { for } s \in(\sigma, 1) \\
z(\sigma)=x
\end{array}\right.
$$

and with $\mathcal{F}$ and $\left(\mathbb{K}_{\theta}\right)_{\theta}$ are satisfying:

(HF1) $\mathcal{F}:(0,1) \times \mathbb{R}^{d} \times A \rightarrow \mathbb{R}^{d}$ is measurable with respect to the time variable, and is continuous with respect to the last two variables $z$ and a. Moreover, for each $(z, a) \in \mathbb{R}^{d} \times A$, we have $\mathcal{F}(\cdot, z, a) \in L^{1}(0,1)$, and $\mathcal{F}(t, z, \mathcal{A})$ is nonempty compact and convex set, for every $x \in \mathbb{R}^{d}$ and for almost every $t \in(0,1)$.

(HF2) There exists $k_{0}>0$ such that $\forall s \in(0,1), x, z \in \mathbb{R}^{d}, a \in A$, we have

$$
|\mathcal{F}(s, x, a)-\mathcal{F}(s, z, a)| \leq k_{0}|x-z|, \quad|\mathcal{F}(t, z, a)| \leq k_{0} .
$$

(HK) the set-valued application $\theta \rightsquigarrow \mathbb{K}_{\theta}$ is upper semicontinuous on $[0,1]$. 
Our goal is to characterize the new value function $\vartheta$. It is easy to check that the corresponding control problem does not satisfy any controllability condition. Indeed, the field $\mathcal{F}$ can never be inward pointing (resp. outward pointing) on $\bigcup_{s \in[0,1]} \times \mathbb{K}(s)$. Then the characterization of $\vartheta$ as constrained viscosity solution of an HJB equation does not hold in the general case [4].

4.1. Epigraph of $\vartheta$. First of all, to deal with the state constraints, we introduce a Lipschitz continuous function $\Psi:[0,1] \times \mathbb{R}^{d} \rightarrow \mathbb{R}$ such that

$$
\Psi(\theta, x) \leq 0 \Leftrightarrow x \in \mathbb{K}_{\theta}, \forall \theta \in[0,1], x \in \mathbb{R}^{d} .
$$

(Note that this is always possible to find such a function $\Psi$. In particular, according to theorem 3.2, the distance function to the set $\bigcup_{\theta \in[0,1]}\{\theta\} \times \mathbb{K}_{\theta}$ fulfilled the conditions). By using an idea introduced in [1], an equivalent way to characterize the epigraph of $\vartheta$ consists of considering the control problem

$$
\left.w(\sigma, x, \xi)=\inf _{\substack{\alpha \in \mathcal{A} \\ \zeta=0, \zeta(\sigma)=\xi}}\left\{\left(\varphi\left(z_{x, \sigma}^{\alpha}(1)\right)-\zeta(1)\right) \bigvee \max _{\theta \in[\sigma, 1]} \Psi\left(\theta, y_{x, \sigma}^{\alpha}(\theta)\right)\right)\right\}
$$

where now, the state constraints are included in the cost function to be minimized. In the above expression the notation $a \vee b$ means the $\max (a, b)$. The following result shows the relation between the 0 -level set of $w$ and the epigraph of $\vartheta$ :

Proposition 4.2. Assume that (Hg1)-(Hg2) and $(\boldsymbol{H} \varphi)$ hold true, then we have

$$
\begin{aligned}
\left\{(\sigma, x, z) \in[0,1] \times \mathbb{R}^{d} \times \mathbb{R} \mid w(\sigma, x, z) \leq 0\right\} & =\left\{(\sigma, x, z) \in[0,1] \times \mathbb{R}^{d} \times \mathbb{R} \mid \vartheta(\sigma, x) \leq z\right\} \\
& =: \mathcal{E} p \vartheta
\end{aligned}
$$

and $v(\sigma, x)=\min \{z \mid w(\sigma, x, z) \leq 0\}$.

Proof. First, let us point out that under assumptions ( $\mathrm{Hg} 1)-(\mathrm{Hg} 2)$, by Filippov theorem, the set of trajectories $S_{[\sigma, 1]}^{P}(x)$ is compact in $C([\sigma, 1])$, then the infimum in the definition of $w$ is achieved. Moreover, when $\vartheta$ is finite, the infimum in the definition of $\vartheta$ is achieved too. Let $(\sigma, x, \xi)$ be in $[0,1] \times \mathbb{R}^{d} \times \mathbb{R}$, it comes that:

$$
\begin{aligned}
w(\sigma, x, \zeta) \leq 0 & \Leftrightarrow \exists \alpha \in \mathcal{A} \text { s.t. } \varphi\left(z_{x, \sigma}^{\alpha}(1)\right) \leq \zeta, \Psi\left(\theta, z_{x, \sigma}^{\alpha}(\theta)\right) \leq 0, \forall \theta \in[\sigma, 1] \\
& \Leftrightarrow \exists \alpha \in \mathcal{A} \text { s.t. } \varphi\left(z_{x, \sigma}^{\alpha}(1)\right) \leq \zeta, z_{x, \sigma}^{\alpha}(\theta) \in \mathbb{K}(\theta), \forall \theta \in[\sigma, 1] \\
& \Leftrightarrow \vartheta(\sigma, x) \leq \zeta .
\end{aligned}
$$

Proposition 4.2 shows that once the auxiliary function $w$ is computed, the epigraph of $\vartheta$ can be deduced as the 0 -level set of $w$.

4.2. Characterization of $w$. Hence, the goal now is to characterize the auxiliary function $w$. As in the classical case, the function $w$ can be characterized as the unique solution of a Hamilton-Jacobi equation. More precisely, considering the Hamiltonian

$$
\mathcal{H}(\sigma, x, p):=\sup _{a \in A}(-\mathcal{F}(\sigma, x, a) \cdot p)
$$

we have 
TheOrem 4.3. Assume that (Hg1)-(Hg2), $(\boldsymbol{H} \varphi)$ hold and that $\mathcal{K}$ is a closed nonempty set of $\mathbb{R}^{d}$. Then $w$ is the unique continuous viscosity solution of the variational inequality

$$
\min \left(-\partial_{s} w(\sigma, x, \xi)+\mathcal{H}(\sigma, x, D w), w(\sigma, x, \xi)-\Psi(\sigma, x)\right)=0
$$

for $s \in(0,1),(x, \xi) \in \mathbb{R}^{d+1}$, and

$$
w(1, x, \xi)=\max (\varphi(x)-\xi, \Psi(1, x)), x, \xi \in \mathbb{R}^{d+1} .
$$

As usual, the proof of Theorem 4.3 is based on the dynamic programming principle (DPP) satisfied by $w$, and that can be stated here as follows:

Lemma 4.4. The function $w$ is characterized by

1. for all $t \in[0,1]$ and $\tau \in[0,1-t]$, for all $x, \xi \in \mathbb{R}^{d+1}$,

$$
w(t, x, \xi)=\inf _{\alpha \in \mathcal{A}}\left\{w\left(t+\tau, z_{x, t}^{\alpha}(t+\tau), \xi\right) \bigvee \max _{\theta \in[t, t+\tau]} \Psi\left(\theta, z_{x, t}^{\alpha}(\theta)\right)\right\}
$$

2. $w(1, x, \xi)=\max (\varphi(x)-\xi, \Psi(1, x)),(x, \xi) \in \mathbb{R}^{d+1}$.

The first consequence of the above lemma is the continuity of the value function $w:$

Proposition 4.5. Assume $(\boldsymbol{H g} \mathbf{1})-(\boldsymbol{H g} 2)$ and $(\boldsymbol{H} \varphi)$ hold, and $\mathcal{K}$ is a closed set of $\mathbb{R}^{d}$. Then $w$ is Lipschitz continuous on $[0,1] \times \mathbb{R}^{d+1}$.

Proof. Let $t \in[0,1]$ and $(x, \xi),\left(x^{\prime}, \xi^{\prime}\right) \in \mathbb{R}^{n+1}$. By using the definition of $w$ and the simple inequalities:

$$
\begin{array}{r}
\max (A, B)-\max (C, D) \leq \max (A-C, B-D), \\
\inf A_{\alpha}-\inf B_{\alpha} \leq \sup \left(A_{\alpha}-B_{\alpha}\right),
\end{array}
$$

we get:

$$
\begin{aligned}
& \left|w(t, x, \xi)-w\left(t, x^{\prime}, \xi^{\prime}\right)\right| \\
\leq & \sup _{\alpha \in \mathcal{A}} \max \left(\left|\varphi\left(z_{x, t}^{\alpha}(1)\right)-\varphi\left(z_{x^{\prime}, t}^{\alpha}(1)\right)\right|+\left|\xi-\xi^{\prime}\right|, \max _{\theta \in[t, 1]}\left|\Psi\left(\theta, z_{x, t}^{\alpha}(\theta)\right)-\Psi\left(\theta, z_{x^{\prime}, t}^{\alpha}(\theta)\right)\right|\right) \\
\leq & \sup _{\alpha \in \mathcal{A}}\left(m_{\varphi}\left\|z_{x, t}^{\alpha}(1)-z_{x^{\prime}, t}^{\alpha}(1)\right\|+\left|\xi-\xi^{\prime}\right|, \max _{\theta \in[t, 1]}\left\|z_{x, t}^{\alpha}(\theta)-z_{x^{\prime}, t}^{\alpha}(\theta)\right\|\right),
\end{aligned}
$$

where $m_{\Phi}$ is the Lipschitz constant of $\varphi$. By assumption $(\mathbf{H g} \mathbf{1})-(\mathbf{H g} \mathbf{2})$, we know that $\left\|z_{x, t}^{\alpha}(\theta)-z_{x^{\prime}, t}^{\alpha}(\theta)\right\| \leq e^{k_{0}}\left\|x-x^{\prime}\right\|$ for all $\alpha \in \mathcal{A}, \theta \in[t, 1]$, then we conclude that:

$$
\left|w(t, x, \xi)-w\left(t, x^{\prime}, \xi^{\prime}\right)\right| \leq \max \left(m_{\Phi} e^{k_{0}}\left\|x-x^{\prime}\right\|+\left\|\xi-\xi^{\prime} \mid, e^{k_{0}}\right\| x-x^{\prime} \|\right),
$$

and we deduce that $w(t, \cdot, \cdot)$ is Lipschitz continuous in $\mathbb{R}^{d} \times \mathbb{R}$. Now let $(x, \xi) \in \mathbb{R}^{d+1}$ and $t \in[0,1], \tau \in[0,1-t]$. Remarking that $w(t+\tau, x, \xi) \geq \Psi(t+\tau, x, \xi)$ and by using 
the DPP, it follows that:

$$
\begin{aligned}
|w(t, x, \xi)-w(t+\tau, x, \xi)|= & \mid \inf _{\alpha \in \mathcal{A}} \max \left(w\left(t+\tau, z_{x, t}^{\alpha}(t+\tau), \xi\right), \max _{\theta \in[t, t+\tau]} \Psi\left(\theta, z_{x, t}^{\alpha}(\theta)\right)\right) \\
& -\max (w(t+\tau, x, \xi), g(t+\tau, x)) \mid \\
\leq & \sup _{\alpha \in \mathcal{A}} \max \left(\left|w\left(t+\tau, z_{x, t}^{\alpha}(t+\tau), \xi\right)-w(t+\tau, x, \xi)\right|,\right. \\
& \left.\left|\max _{\theta \in[t, t+\tau]} \Psi\left(\theta, z_{x, t}^{\alpha}(\theta)\right)-\Psi(t+\tau, x)\right|\right) \\
\leq & \max \left(m_{\varphi}\left(e^{k_{0}} k_{0} \tau\right), e^{k_{0}} k_{0} \tau,\left(1+k_{0}\right) \tau\right)
\end{aligned}
$$

where we have used (4.4) and assumptions (Hg1)-(Hg2). This completes the proof. [

Before proving the theorem 4.3, once need first to make more precise the notion of $L^{1}$-viscosity solution for (4.2). Here we extend the $L^{1}$-viscosity notion introduced by Ishii in[23].

Definition 4.6. A lower semi-continuous (resp. upper semi-continuous) function $u:(0,1) \times \mathbb{R}^{d} \times \mathbb{R} \rightarrow \mathbb{R}$ is a $L^{1}$-viscosity supersolution (resp. subsolution) of (4.2) if

1. $u(1, x, \xi) \geq(\varphi(x)-\xi) \bigvee \Psi(1, x)($ resp. $u(1, x, \xi) \leq(\varphi(x)-\xi) \bigvee \Psi(1, x))$;

2. For any test function $b \in L^{1}(0,1), \phi \in C^{1}\left(\mathbb{R}^{d+1}\right)$ such that $u(t, x, \xi)-$ $\int_{0}^{1} b(s) d s-\phi(x, \xi)$ achieves a local minimum (resp. maximum) on $\left(t_{0}, x_{0}, \xi_{0}\right) \in$ $(0,1) \times \mathbb{R}^{n+1}$, we have

$$
\begin{gathered}
\min \left(\lim _{\delta \rightarrow 0^{+}} \operatorname{ess} \sup _{\left|t-t_{0}\right| \leq \delta} \sup _{\substack{(x, \xi) \in B_{\delta}\left(x_{0}, \xi_{0}\right), p \in B_{\delta}\left(D \phi\left(\mathcal{S}_{0}, \xi_{0}\right)\right)}}\{\mathcal{H}(t, x, p)-b(t)\},(u-\Psi)\left(t_{0}, x_{0}\right)\right) \geq 0 \\
\text { (resp. } \left.\min \left(\lim _{\delta \rightarrow 0^{+}} \operatorname{essinf}_{\left|t-t_{0}\right| \leq \delta} \inf _{\substack{(x, \xi) \in B_{\delta}\left(x_{0}, \xi_{0}\right), p \in B_{\delta}\left(D \phi\left(x_{0}, \xi_{0}\right)\right)}}\left\{\mathcal{H}\left(t, x_{0}, p\right)-b(t)\right\},(u-\Psi)\left(t_{0}, x_{0}\right)\right) \leq 0\right) .
\end{gathered}
$$

A continuous function $u$ is a $L^{1}$-viscosity solution of (4.2) if $u$ is both a supersolution and a subsolution of (4.2).

Now, we can give the proof of Theorem 4.3.

Proof. [Proof of Theorem 4.3] We first show that $w$ is a solution of (4.2). The fact that $w$ satisfies the initial condition is a direct consequence of Lemma 4.4(ii). Let us check the $L^{1}$-supersolution property of $w$. By the definition of $w$, for every $(\sigma, x, \xi) \in[0,1] \times \mathbb{R}^{d} \times \mathbb{R}$, we have

$$
w(\sigma, x, \xi) \geq \inf _{\alpha \in \mathcal{A}} \max _{\theta \in[\sigma, 1]} \Psi\left(\theta, z_{x, \sigma}^{\alpha}(\theta)\right) \geq \Psi(\sigma, x) .
$$

Let $b \in L^{1}(0,1), \phi \in C^{1}\left(\mathbb{R}^{n+1}\right)$ and $\left(\sigma_{0}, x_{0}, \xi_{0}\right) \in(0,1) \times \mathbb{R}^{n+1}$ be a local minimum point of $w(\sigma, x, \xi)-\int_{0}^{1} b(s) d s-\phi(x, \xi)$, then there exists $\delta>0$ such that

$$
w(\sigma, x, \xi)-\int_{0}^{\sigma} b(s) d s-\phi(x, \xi) \geq w\left(\sigma_{0}, x_{0}, \xi_{0}\right)-\int_{0}^{\sigma_{0}} b(s) d s-\phi\left(x_{0}, \xi_{0}\right),
$$

for any $\sigma \in\left[\sigma_{0}-\delta, \sigma_{0}+\delta\right],(x, \xi) \in B_{\delta}\left(x_{0}, \xi_{0}\right)$. By Lemma 4.4(i), for all $\varepsilon>0$, there exists $\alpha_{0} \in \mathcal{A}$ such that

$$
w\left(\sigma_{0}, x_{0}, \xi_{0}\right) \geq w\left(\sigma_{0}+\tau, z_{x_{0}, \sigma_{0}}^{\alpha_{0}}\left(\sigma_{0}+\tau\right), \xi_{0}\right)-\varepsilon, \forall \tau \in\left[0,1-\sigma_{0}\right] .
$$


Consider some $\tau \leq \delta$, then by (4.6) and (4.7), we have

$$
\int_{0}^{\sigma_{0}} b(s) d s+\phi\left(x_{0}, \xi_{0}\right) \geq \int_{0}^{\sigma_{0}+\tau} b(s) d s+\phi\left(z_{x_{0}, \sigma_{0}}^{\alpha_{0}}\left(\sigma_{0}+\tau\right)\right)-\varepsilon,
$$

i.e.

$$
-\int_{\sigma_{0}}^{\sigma_{0}+\tau}\left[D \phi\left(z_{x_{0}, \sigma_{0}}^{\alpha_{0}}(s)\right) \cdot \mathcal{F}\left(s, z_{x_{0}, \sigma_{0}}^{\alpha_{0}}(s), a_{0}(s)\right)+b(s)\right] d s \geq-\varepsilon
$$

then by the definition of the Hamiltonian, we have

$$
\int_{\sigma_{0}}^{\sigma_{0}+\tau}\left[\mathcal{H}\left(s, z_{z_{0}, \sigma_{0}}^{\alpha_{0}}(s), D \phi\left(z_{x_{0}, \sigma_{0}}^{\alpha_{0}}(s)\right)\right)-b(s)\right] d s \geq-\varepsilon, \forall \varepsilon>0,
$$

and we deduce that

$$
\int_{\sigma_{0}}^{\sigma_{0}+\tau}\left[\mathcal{H}\left(s, z_{x_{0}, \sigma_{0}}^{\alpha_{0}}(s), D \phi\left(z_{x_{0}, \sigma_{0}}^{\alpha_{0}}(s)\right)\right)-b(s)\right] d s \geq 0 .
$$

By contradiction, we assume that

$$
\lim _{\delta \rightarrow 0^{+}} \operatorname{ess} \sup _{\left|\sigma-\sigma_{0}\right| \leq \delta} \sup _{\substack{(x, \xi) \in B_{\delta}\left(x_{0}, \xi_{0}\right), p \in B_{\delta}\left(D \phi\left(x_{0}, \xi_{0}\right)\right)}}\{\mathcal{H}(\sigma, x, \xi, p)-b(t)\}<0,
$$

then there exists $\delta_{1}>0, E \subset\left[\sigma_{0}-\delta_{1}, \sigma_{0}+\delta_{1}\right]$ with $m(E)=0$ such that $\forall s \in$ $\left[\sigma_{0}-\delta_{1}, \sigma_{0}+\delta_{1}\right] \backslash E,(x, \xi) \in B_{\delta_{1}}\left(x_{0}, \xi_{0}\right)$ and $p \in B_{\delta_{1}}\left(D \phi\left(x_{0}, \xi_{0}\right)\right)$, we have $\mathcal{H}(s, x, p)$ $b(s)<0$. By the continuity of $z_{x_{0}, \sigma_{0}}^{\alpha}(\cdot), D \phi(\cdot)$ and $\mathcal{H}(t, \cdot, \cdot)$, for $\tau$ small enough, we get

$$
H\left(s, z_{x_{0}, \sigma_{0}}^{\alpha}(s), D \phi\left(z_{x_{0}, t_{0}}^{\alpha}(s), \xi_{0}\right)\right)-b(s)<0, \text { for } s \in\left[t_{0}, t_{0}+\tau\right] \backslash E,
$$

which contradicts (4.8). Combined with (4.5), we get

$\min \left(\lim _{\delta \rightarrow 0^{+}} \operatorname{ess} \sup _{\left|\sigma-\sigma_{0}\right| \leq \delta} \sup _{(x, \xi) \in B_{\delta}\left(x_{0}, \xi\right), p \in B_{\delta}\left(D \phi\left(x_{0}, \xi_{0}\right)\right.}\{\mathcal{H}(\sigma, x, p)-b(\sigma)\}, w\left(\sigma_{0}, x_{0}, \xi_{0}\right)-\Psi\left(\sigma_{0}, x_{0}\right)\right) \geq 0$.

Let us now prove that $w$ is a $L^{1}$-subsolution. Let $\left(\sigma_{0}, x_{0}, \xi_{0}\right) \in(0,1) \times \mathbb{R}^{d+1}$. If $w\left(\sigma_{0}, x_{0}, \xi_{0}\right) \leq \Psi\left(\sigma_{0}, x_{0}\right)$, it is obvious that $w$ satisfies

$$
\min \left(-\partial_{t} w\left(\sigma_{0}, x_{0}, \xi_{0}\right)+\mathcal{H}\left(\sigma_{0}, x_{0}, D w\left(t_{0}, x_{0}, \xi_{0}\right)\right), w\left(\sigma_{0}, x_{0}, \xi_{0}\right)-\Psi\left(t_{0}, x_{0}, \xi_{0}\right)\right) \leq 0
$$

in the $L^{1}$-viscosity sense. Now, assume that $w\left(t_{0}, x_{0}, \xi_{0}\right)>\Psi\left(t_{0}, x_{0}, \xi_{0}\right)$. By continuity of $w$ and $\Psi$, there exists some $\tau>0$ such that $w\left(\sigma_{0}+\tau, z_{x_{0}, \sigma_{0}}^{\alpha}\left(\sigma_{0}+\tau\right)\right)>\Psi\left(\theta, z_{x_{0}, t_{0}}^{\alpha}(\theta)\right)$ for all $\theta \in\left[\sigma_{0}, \sigma_{0}+\tau\right]$ ( since $z_{x_{0}, \sigma_{0}}^{\alpha}(\theta)$ will stay in a neighborhood of $x_{0}$ which is controlled uniformly with respect to $a$ ). Hence, by using Lemma 4.4(i), we get that

$$
w\left(\sigma_{0}, x_{0}, \xi_{0}\right)=\inf _{\alpha \in \mathcal{A}} w\left(\sigma_{0}+h, z_{x_{0}, t_{0}}^{\alpha}\left(t_{0}+h\right)\right), \forall h \in[0, \tau]
$$

We then deduce by the same argument as for the supersolution property that $-\partial_{t} w\left(\sigma_{0}, x_{0}, \xi_{0}\right)+$ $\mathcal{H}\left(\sigma_{0}, x_{0}, D w\left(\sigma_{0}, x_{0}, \xi_{0}\right)\right) \leq 0$ in the $L^{1}$-viscosity sense. Therefore, $w$ is a $L^{1}$-viscosity subsolution.

The uniqueness follows from the following comparison principle result. 
Proposition 4.7 (Comparison principle). If $u$ is a $L^{1}$-viscosity subsolution and $v$ is a $L^{1}$-viscosity supersolution of (4.2), then we have

$$
u \leq v \text {, on }(0,1) \times \mathbb{R}^{d+1} \text {. }
$$

Proof. By Definition 4.6, for any $(t, x, \xi) \in(0,1) \times \mathbb{R}^{d+1}$ we have that

$$
\begin{gathered}
\min \left(-\partial_{t} u(t, x, \xi)+H(t, x, D u), u(t, x, \xi)-\Psi(t, x)\right) \leq 0 \\
\min \left(-\partial_{t} v(t, x, \xi)+H(t, x, D v), v(t, x, \xi)-\Psi(t, x)\right) \geq 0
\end{gathered}
$$

in the $L^{1}$-viscosity sense. If $u(t, x, \xi)-\Psi(t, x) \leq 0$, we get

$$
u(t, x, \xi) \leq \Psi(t, x) \leq v(t, x, \xi) .
$$

If $u(t, x, \xi)-\Psi(t, x)>0$, then we have

$$
-\partial_{t} u(t, x, \xi)+H(t, x, D u) \leq 0, \quad-\partial_{t} v(t, x, \xi)+H(t, x, D v) \geq 0,
$$

where we get $u(t, x, \xi) \leq v(t, x, \xi)$ from a classical comparison principle (see Theorem 8.1 in Ishii[23]).

\section{REFERENCES}

[1] A. Altarovici, O. Bokanowski, and H. Zidani. A general Hamilton-Jacobi framework for nonlinear state-constrained control problems. http://hal.inria.fr/hal-00653337/fr/, 2011.

[2] A. Arutyunov, V. Dykhta, and L. Lobo Pereira. Necessary conditions for impulsive nonlinear optimal control problems without a priori normality assumptions. Journal of Optimization Theory and applications, 124(1):55-77, 2005.

[3] J. Bechhofer and B. Johnson. A simple model for Faraday waves. Amer. J. Phys., 64:1482-1487, 1996.

[4] O. Bokanowski, N. Forcadel, and H. Zidani. Deterministic state constrained optimal control problems without controllability assumptions. ESAIM: Control, Optimisation and Calculus of Variations, 17(04):975-994, 2011.

[5] R.M Brach. Mechanical Impact Dynamics. John Wiley, New York, 1991.

[6] A. Bressan. Impulsive control systems. In B. Mordukhovich and H. Sussmann, editors, Nonsmooth Analysis and Geometric Methods in deterministic Optimal Control, pages 1-22. Springer, New York, 1996.

[7] A. Bressan and F. Rampazzo. On differential systems with vector-valued impulsive controls. Boll.Un.Mat.Ital., 7(2-B):641-656, 1988.

[8] A. Bressan and F. Rampazzo. Impulsive control-systems with commutativity assumptions. Journal of optimization theory and applications, 71(1):67-83, 1991.

[9] A. Bressan and F. Rampazzo. Impulsive control-systems without commutativity assumptions. Journal of optimization theory and applications, 81(3):435-457, 1994.

[10] A. Briani. A Hamilton-Jacobi equation with measures arising in $\Gamma$-convergence of optimal control problems. Differential and Integral Equations, 12(6):849-886, 1999.

[11] A. Briani and H. Zidani. Characterisation of the value function of final state constrained control problems with bv trajectories. Communication in Pure and Applied Analysis, 10(6):15671587, 2011.

[12] B. Brogliato. Nonsmooth impact Mechanics: Models, Dynamics and control, volume 220 of Lecture Notes in Control and Information Sciences. Springer Verlag, New York, 1996.

[13] I. Capuzzo-Dolcetta and P.-L. Lions. Hamilton-Jacobi equations with state constraints. Trans. Amer. Math. Soc., 318(2):643-683, 1990.

[14] A. Catllá, J. Porter, and M. Silber. Weakly nonlinear analysis of impulsively-forced Faraday waves. Phys. Rev. E, 72(3), 2005.

[15] A. Catllá, D. Schaeffer, T. Witelski, E. Monson, and A. Lin. On spiking models for synaptic activity and impulsive differential equations. SIAM Review, 50(3):553-569, 2005.

[16] G. Dal Maso and F. Rampazzo. On systems of ordinary differential equations with measures as controls. Differential and Integral Equations, 4(4):738-765, 1991. 
[17] N. Forcadel, Z. Rao, and H. Zidani. Optimal control problems of bv trajectories with pointwise state constraints. In Proceedings of the 18th IFAC World Congress, Milan, volume 18, 2011.

[18] H. Frankowska and S. Plaskacz. Semicontinuous solutions of Hamilton-Jacobi-Bellman equations with degenerate state constraints. J. Math. Anal. Appl., 251(2):818-838, 2000.

[19] H. Frankowska, S. Plaskacz, and T. Rzeuchowski. Measurable viability theorems and HamiltonJacobi-Bellman equation. J. Diff. Eqs, 116:265-305, 1995.

[20] J. Guckenheimer and P. Holmes. Nonlinear oscillations, Dynamical systems, and bifurcations of vector fields, volume 42. Springer-Verlag, New York, 1990.

[21] C. Hsu and W. Cheng. applications of the theory of impulsive parametric excitation excitation and new treatments of general parametric excitation problems. Trans. ASME J. Appl. Mech., 40:551-558, 1973.

[22] C. Huepe, Y. Ding, P. Umbanhowar, and M. Silber. Forcing function control of Faraday wave instabilities in viscous shallow fluids. Phys. Rev. E, 73:-, 2006.

[23] H. Ishii. Hamilton-Jacobi equations with discontinuous Hamiltonians on arbitrary open sets. Bull. Facul. Sci. \& Eng., 28:33-77, 1985.

[24] M. Motta and F. Rampazzo. Dynamic programming for nonlinear systems driven by ordinary and impulsive controls. SIAM J. Control and Optim., 44(1):199-225, 1996.

[25] J.-P. Raymond. Optimal control problems in spaces of functions of bounded variation. Differential Integral Equations, 10:105-136, 1997.

[26] G.N. Silva and R.B. Vinter. Measure-driven differential inclusions. J. of Math. Anal. and Appl., 202:746-767, 1996.

[27] G.N. Silva and R.B. Vinter. Necessary conditions for optimal impulsive control systems. SIAM J. of Control and Optim., 35:1829-1846, 1998.

[28] H. M. Soner. Optimal control with state-space constraint. I. SIAM J. Control Optim., 24(3):552-561, 1986.

[29] H. M. Soner. Optimal control with state-space constraint. II. SIAM J. Control Optim., 24(6):1110-1122, 1986.

[30] P.R. Wolenski and S. Žabić. A differential solution concept for impulsive systems. Differential Equations and Dynamical Systems, 2:199-210, 2006.

[31] P.R. Wolenski and S. Žabić. A sampling method and approximations results for impulsive systems. SIAM J. Control Optim., 46:983-998, 2007. 